\title{
MODELAGEM DA INTERCEPTAÇÃO NA FLORESTA NACIONAL DE CAXIUANÃ, NO LESTE DA AMAZÔNIA.
}

\author{
LEIDIANE LEÃO DE OLIVEIRA ${ }^{1}$, RAFAEL FERREIRA DA COSTA ${ }^{2}$, ANTONIO CARLOS LÔLA DA \\ COSTA $^{3}$, FRANCISCO DE ASSIS SALVIANO DE SOUSA ${ }^{2}$, ALAN PANTOJA BRAGA ${ }^{4}$.
}

\author{
${ }^{1}$ Universidade Federal do Amapá - UNIFAP - PPGBIO, \\ ${ }^{2}$ Universidade Federal de Campina Grande - UFCG - UACA, \\ ${ }^{3}$ Universidade Federal do Pará UFPA - DM, \\ ${ }^{4}$ Instituto Nacional de Meteorologia - INMET. \\ leidiane.leao@iepa.ap.gov.br
}

Recebido Março 2007 - Aceito Dezembro 2007

\begin{abstract}
RESUMO
O presente trabalho foi realizado na Estação Cientifica Ferreira Penna, na Floresta Nacional de Caxiuanã, Melgaço, Pará, Brasil (01 42' 30"S; 51 31' 45”W; 60 m altitude) no Leste da Amazônia. É uma área com floresta de terra firme, vegetação densa e dossel com altura média de $35 \mathrm{~m}$ e algumas árvores emergentes acima de $50 \mathrm{~m}$, e densidade variando de 450 a 550 árvores por hectare. Os objetivos foram de quantificar as estimativas da interceptação por modelagens numéricas, no período de março a dezembro de 2004. A interceptação obtida nas medidas de campo foi de $248 \mathrm{~mm}$, correspondendo a $21,5 \%$ da precipitação total incidente acima do dossel de $1.153,4 \mathrm{~mm}$. As estimativas de interceptação foram simuladas com bom nível de eficiência, utilizando os modelos de Rutter e Gash. O modelo de Gash superestimou a interceptação em 17,3\% $(42,8 \mathrm{~mm})$ do total acumulado, enquanto o modelo de Rutter superestimou em apenas $0,5 \%(1,1 \mathrm{~mm})$ do total acumulado da interceptação.

Palavras-chave: Caxiuanã, Floresta Tropical, Interceptação, Modelos de Gash e de Rutter
\end{abstract}

\begin{abstract}
MODELLING OF INTERCEPTION BY CAXIUANÃ NATIONAL FOREST, IN THE EASTERN AMAZONIA.

The present work was carried out in the Ferreira Penna Scientific Station, at the Caxiuanã National Forest in Melgaço, Pará, Brazil ( $01^{\circ} 42^{\prime} 30^{\prime \prime}$ S; 51 31 31 45” W; 60 m of altitude) in the Eastern Amazonia. It is an upland forest area with closed vegetation, $35 \mathrm{~m}$ average canopy high, some emergent trees with $50 \mathrm{~m}$ high and species density between 450 and 550 plants per hectare. The objective was to compare the estimations of rainfall interception using two numerical models, for the period from March to December of 2004. The measured rainfall interception was $248 \mathrm{~mm}$ corresponding to $21.5 \%$ of total precipitation $(1,153.4 \mathrm{~mm})$ above canopy. The rainfall interception was simulated, by Rutter's and Gash's models, at good level of accuracy. The Gash model overestimated the interception by $17.3 \%(42.8 \mathrm{~mm})$, while the Rutter model overestimated by only $0.5 \%(1.1 \mathrm{~mm})$ of the observed interception.
\end{abstract}

Keywords: Caxiuanã, Gash and Rutter models, Interception, Tropical rainforest

\section{INTRODUÇÃO}

A modelagem numérica e as medidas reais feitas no campo são duas técnicas usadas para se obter a interceptação. A utilização de valores observados experimentalmente é mais confiável, porém, há sérias dificuldades técnicas e financeiras para se instalar um experimento desta natureza, principalmente em áreas florestais. Por esta razão a utilização de modelos é freqüentemente necessária para se quantificar a interceptação.
O ciclo hidrológico tem diversas componentes, porém uma delas é comumente desprezada, a interceptação da precipitação pela vegetação (I). A $I$ tem grande importância no balanço hídrico, principalmente em áreas com florestas de grande porte. Para Nobre et al. (1991) as evidências observacionais disponíveis sugerem que a Amazônia é altamente eficiente na reciclagem da água na atmosfera, e a substituição da floresta por pastagens não manteria a elevada taxa de evapotranspiração. Eltahir e Bras (1994) estimaram através 
de um modelo de reciclagem da precipitação que entre $25 \%$ e $35 \%$ da precipitação ocorrida na bacia amazônica seriam provenientes da evapotranspiração local.

A influência da vegetação no recebimento e redistribuição das chuvas é significativa dentro do contexto do balanço hídrico de um determinado local. Em alguns casos é comum se confundir a quantidade de água precipitada acima do dossel, com a água realmente disponível para o solo. A falta de informação de I pode induzir a erros nas medidas reais das quantidades de água que contribuirão para a reposição da umidade disponível no solo, comprometendo o cálculo do balanço hídrico.

Entre os trabalhos sobre interceptação $(I)$ destaca-se o de Horton (1919). Em seu estudo quase secular o autor apresentou as bases para a modelagem numérica da $I$, considerando que a interceptação seria o resultado da soma da capacidade de armazenamento de água pelo dossel vegetal com o produto das taxas de evaporação durante a chuva, o tempo de duração dessa chuva em horas, e a razão de evaporação da superfície plana projetada pela copa da árvore no solo. Nesse seu estudo, Horton descreveu que a $I$ varia entre 15 e $80 \%$ da precipitação total medida em área aberta ou acima da vegetação. Essa variação depende de diversos fatores das diferentes espécies vegetais, tais como; idade da floresta e densidade de exemplares na área de estudo, índice de área foliar (IAF) e características da precipitação, entre outros.

Rutter et al., (1971 e 1975) apresentaram um modelo fisicamente fundamentado baseado em dados horários, sendo que este surgiu como sendo o modelo mais apropriado para descrever o processo de $I$, e tem sido aplicado em diversos tipos de florestas. É executado através da entrada de variáveis controladoras do processo de evaporação e parâmetros morfológicos da vegetação. Gash (1979) testou e descreveu o modelo de Rutter como sendo o mais confiável para se estimar os valores interceptados. Nesse estudo foram considerados termos negligenciados por Rutter et al. (1971 e 1975), tais como, a evaporação do dossel após o término da chuva, os efeitos das chuvas de pequena intensidade que não são capazes de saturar o dossel mais o deixam úmido, e a evaporação dos troncos das árvores.

O presente estudo teve o objetivo de aplicar os modelos numéricos de Rutter e de Gash, para a estimativa da I na Floresta Nacional (FLONA) de Caxiuanã, Pará, Brasil.

\section{MATERIAL E MÉTODOS}

\section{1. Área de estudo}

O estudo foi desenvolvido em uma floresta tropical úmida de terra firme que está localizada na Floresta Nacional (FLONA) de Caxiuanã, no município de Melgaço, Pará, onde funciona a Estação Científica Ferreira Penna (ECFPn) $\left(01^{\circ}\right.$
42’ 30”S; 51 31' 45”'W; $62 \mathrm{~m}$ de altitude), uma descrição detalhada da área experimental está em Oliveira (2007).

A região da FLONA de Caxiuanã tem uma distribuição regular das chuvas durante todo o ano. Porém, há uma sazonalidade definida. Climatologicamente, o período chuvoso está compreendido de dezembro a junho, enquanto o seco vai de agosto a novembro. Para o ano de 2004, o total anual precipitado foi de $2.359,4 \mathrm{~mm}$, cerca de $400 \mathrm{~mm}(20,4 \%)$ acima da média do período de 1996 a 2003 que foi de 1.959,7 mm. Durante o período chuvoso de 2004, ocorreram $1.845 \mathrm{~mm}$, representando $78,2 \%$ das chuvas, enquanto no período seco, o total precipitado foi de $514,4 \mathrm{~mm}$, ou $21,8 \%$ do total anual. O mês mais chuvoso em 2004 foi março com $474 \mathrm{~mm}$, e o seco foi agosto com 40 mm (Figura 1). Para Souza (2003) as precipitações no Leste da Amazônia sofrem as influências dos Oceanos Atlântico e Pacífico no primeiro trimestre do ano, estando estas precipitações acima da média relacionadas com os eventos de La Niña.

A precipitação máxima da região Amazônica ocorre na parte Noroeste, sem estação seca, e com totais mensais acima de $200 \mathrm{~mm}$, na maioria dos meses. Autores como Figueroa e Nobre (1990) e Marengo (1992) sugeriram que esse máximo pode estar associado à forma côncava da cordilheira dos Andes, que produziria convergência da umidade em baixos níveis, naquela sub-região. Outro máximo de precipitação, embora menos acentuado ocorre no litoral Atlântico da Amazônia. Nesse caso, entretanto, notam-se duas estações bem distintas: a seca de setembro a novembro, com precipitação em torno de $50 \mathrm{~mm}$ mês-1, e a chuvosa de fevereiro a maio com totais superiores a $250 \mathrm{~mm}$ mês $^{-1}$, causada pela penetração da ZCIT que vem a se sobrepor às linhas de instabilidade, ondas de Leste, sistemas convectivo de mesoescala e locais, além de outros fatores que contribuem para a precipitação durante o ano todo, nessa sub-região.

O clima da FLONA de Caxiuanã, pela classificação de Köppen é do tipo tropical quente e úmido e subtipo climático "Am" com uma curta estação seca. A temperatura média do ar oscila em torno de $26,7^{\circ} \mathrm{C}$, com mínimos de $22^{\circ} \mathrm{C}$ e máximos $32{ }^{\circ} \mathrm{C}$ (Ferreira da Costa et al., 2003). O número de horas de brilho de luz solar alcança mais de 2.100 horas ano-1, umidade relativa do ar média anual situa-se em torno de $80 \%$. A direção do vento predominante é de nordeste (Moraes et al., 1997).

A diversidade de espécies vegetais está entre 150 a 160 $\mathrm{ha}^{-1}$, e a densidade de indivíduos variando de 450 a 550 árvores $\mathrm{ha}^{-1}$. As espécies predominantes na floresta de terra-firme são a Eschweilera coriacea (Lecythidaceae), Voucapoua americana (Caesalpiniaceae) e Protium pallidum (Burseraceae) (Viana et al., 2003). No estudo de Silva et al. (2003), foram registradas na ECFPn 1.054 espécies pertencentes a 393 gêneros e 102 famílias. As espécies arbóreas (663) são predominantes na ECFPn, representando $62,9 \%$. 


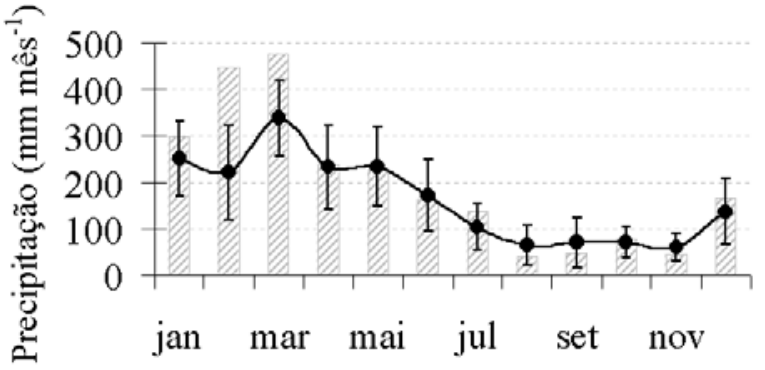

Figura 1 - Precipitação mensal total acima do dossel para o ano de 2004. Os círculos representam as médias mensais (1996-2003) e as barras verticais os desvios-padrão

\subsection{Descrição do experimento}

O presente estudo foi desenvolvido na FLONA de Caxiuanã, Pará, Brasil, no sítio de atividades do experimento ESECAFLOR (O Impacto da Seca Prolongada nos Fluxos de Água e Dióxido de Carbono em uma Floresta Tropical Amazônica), que investiga a influência da exclusão de água do solo sobre o ecossistema de floresta, no ano de 2004.

$\mathrm{O}$ experimento foi realizado de janeiro a dezembro de 2004, período em que foram realizadas 53 coletas semanais. Porém, devido a problemas nos interceptômetros para medidas do escoamento pelos troncos (ESC) somente as informações de 40 coletas foram efetivamente utilizadas para os cálculos da $I$, entre 18 de março e 30 de dezembro.

Foi aplicada a técnica da utilização de uma parcela aproximadamente quadrada. Esse formato da parcela de estudo não foi escolhido de maneira aleatória. Esse padrão permite o fácil acesso ao interior da área sem os conseqüentes danos à vegetação rasteira, além de incluir um grande número de árvores dominantes. Uma área com um hectare representativo da floresta tropical de terra firme foi dividida em subparcelas de $10 \mathrm{~m} \mathrm{X} 10 \mathrm{~m}$, possibilitando cem posições de coleta, seguindo o proposto por Lloyd e Marques Filho (1988).

Em qualquer estudo para determinar $I$, a informação básica necessária para a análise será a precipitação incidente em área aberta ou acima do dossel vegetal (P), que foi a opção escolhida no presente estudo. $P$ foi coletada por um pluviógrafo do tipo CSI Model CS700-L, Rain Gage, com 314,2 cm² de área de captação e capacidade basculante de $100 \mathrm{~mm} \mathrm{~h}^{-1}$, instalado no topo da torre micrometeorológica com $52 \mathrm{~m}$ de altura que se encontra a $2 \mathrm{~km}$ ao Norte da área experimental. Como o dossel médio apresenta árvores entre 30 e $35 \mathrm{~m}$, com alguns exemplares emergentes de até $50 \mathrm{~m}$, as medidas de precipitação não sofreram a influência dos obstáculos vegetais nos arredores.

Para a coleta de dados de Precipitação interna (PI) foram utilizados 25 coletores alternativos, construídos com garrafas "pets" para armazenar 2 litros de água precipitada. Para coleta dessa água foram utilizados funis de alumínio com área de captação de $153,94 \mathrm{~cm},{ }^{2}$ com um suporte de madeira, no qual foi nivelado ficando a $1 \mathrm{~m}$ acima do solo. Esses pluviômetros foram distribuídos aleatoriamente dentro da área experimental, e dispostos nos centros de cada célula com uma distância média entre eles de $10 \mathrm{~m}$. A coleta dos dados foi feita semanalmente com o auxílio de uma proveta graduada em mililitros. Foi utilizado o processo de amostragem aleatória da população, onde os coletores são trocados aleatoriamente após cada coleta, para evitar o viciamento das medidas, possibilitando melhores resultados e eliminando as posições fixas na parcela utilizadas por Helvey e Patrick (1965) apud Ubarana (1994). Toda semana era realizado um sorteio para definir as suas novas posições. Dessa forma, espera-se que com a rotação dos pluviômetros haja uma redução do erro nas medidas de PI. Durante as 40 coletas semanais com informações utilizadas neste trabalho, todas as cem subparcelas possíveis para a localização dos vinte e cinco pluviômetros foram utilizadas.

O ESC é até negligenciado em alguns estudos (Horton, 1919; Lima e Nicolielo, 1983) por significar pequenos percentuais, algo em torno de 1 a $5 \%$ dos totais precipitados. $\mathrm{O}$ ESC tem sua importância no ciclo hidrológico como uma forma eficiente de reposição de água no solo, pois chega à superfície em baixa velocidade devido ao atrito com a casca das árvores, se direcionando próximo às raízes e reduzindo o escoamento superficial. Essa água infiltrará mais facilmente no solo, o que favorecerá o reabastecimento do lençol freático que forma os reservatórios subterrâneos. Para obtenção do ESC a coleta dos dados foi por árvore individual na célula demarcada de $10 \mathrm{mx}$ $10 \mathrm{~m}$, onde foram escolhidas sete árvores com diâmetro à altura do peito (DAP) representativa da parcela, para colocação de anéis coletores (interceptômetros).

\subsection{Modelagem para estimativa da interceptação da precipitação pela vegetação $(I)$}

\subsubsection{Descrição do modelo Rutter}

Foi utilizado o modelo de Rutter (Rutter et al., 1971 e 1975), o qual é fisicamente fundamentado, baseado essencialmente nos cálculos dinâmicos dos balanços hídricos do dossel e dos troncos (Equações 1 e 2, respectivamente). Esse modelo tem sido o mais utilizado para estimativa de $I$ e aplicado em diversos tipos de florestas. O fluxograma do modelo de Rutter (Figura 2) descreve o processo de interceptação (Valente et al., 1997 e Valente, 1999).

$$
\begin{aligned}
& \mathrm{y}_{\mathrm{i}}=\mathrm{e}_{1}+\mathrm{b}_{0} \mathrm{x}_{0}+\mathrm{b}_{1} \mathrm{x}_{1}+\mathrm{b}_{2} \mathrm{x}_{2}+\mathrm{b}_{3} \mathrm{x}_{3 \mathrm{i}}+\mathrm{b}_{4} \mathrm{x}_{4 \mathrm{i}}+\ldots \mathrm{b}_{\mathrm{n}} \mathrm{x}_{\mathrm{ni}} \\
& \mathrm{D}_{\text {previsto }}-\mathrm{D}_{\text {observado }}=\text { Erro }
\end{aligned}
$$


Sendo: $p$ o coeficiente de precipitação livre direta; $p_{\mathrm{t}}$ a parcela de chuva que escoa pelos galhos e tronco; $P$ a precipitação total incidente acima do dossel; E a taxa de evaporação da água interceptada pelo dossel; $D$ a drenagem; $\Delta \mathrm{C}$ a variação da quantidade de água armazenada no dossel; ESC o escoamento pelos troncos; Et a taxa de evaporação da água armazenada nos troncos; $\Delta \mathrm{C}_{\mathrm{t}}$ a variação da quantidade de água armazenada nos troncos. O modelo requer que sejam definidos alguns parâmetros referentes à morfologia da vegetação. Os valores da taxa de aumento da drenagem $(b=5,25)$ com a variação da quantidade de água armazenada no dossel $(C)$ e a drenagem $\left(D_{\mathrm{s}}=0,0014\right)$ quando $(\mathrm{C}=\mathrm{S})$ são os propostos por Lloyd e Marques Filho (1988).

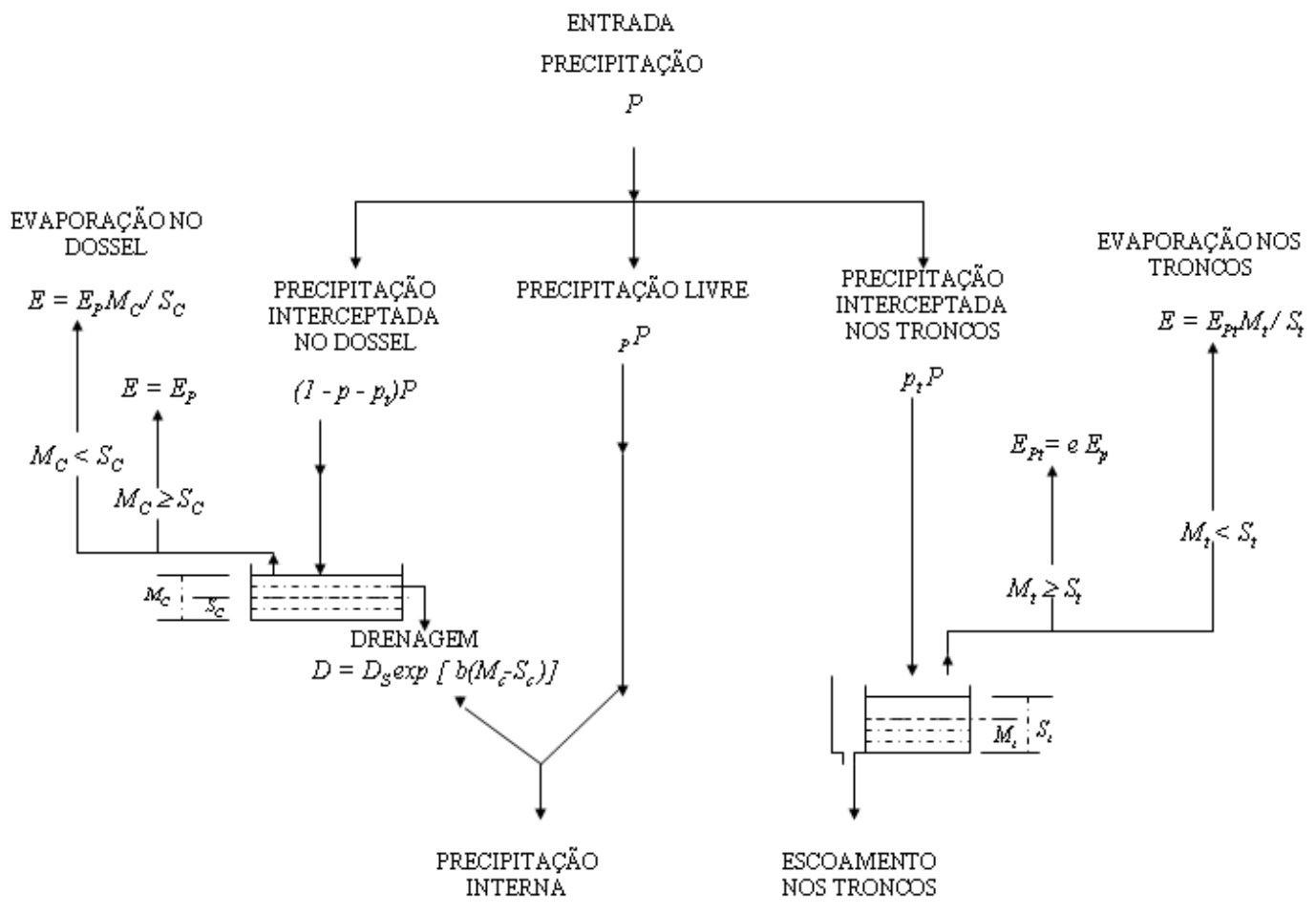

Figura 2 - Fluxograma do modelo de Rutter. Adaptado de (Rutter et al., 1971)

\subsubsection{Descrição do modelo analítico simplificado de Gash}

Estudos de $I$ e $E$ para florestas resultaram freqüentemente em equações empíricas na forma de regressão entre $I$ e $P$, isto é, como funções do tipo:

$$
I=a P+b
$$

Sendo $I$ a quantidade de água interceptada e perdida por evaporação, $\mathrm{P}$ é o total de chuva acima do dossel, e a e b são os coeficientes de regressão. Essa equação pode ser usada para descrever conjuntos de dados de $\mathrm{P}$, ou, se assume que há apenas um evento de $\mathrm{P}$ por dia, para descrever a $I$ diária como uma função da $P$.

Gash (1979) citou o trabalho de Horton (1919) como o primeiro a expressar a I, para grandes eventos de chuva capazes de saturar o dossel. Evidenciando que a evaporação do tronco foi negligenciada.

A taxa média da evaporação, $\bar{E}$, do dossel saturado durante a chuva é definida por:

$$
\bar{E}=\left(\frac{1}{t-t^{\prime}}\right) \int_{t^{\prime}}^{t} E d t
$$

Sendo $\bar{E}$ a taxa média da evaporação do dossel saturado durante a chuva e similarmente é a taxa média da precipitação. Tendo ainda

$$
P-P^{\prime}=\bar{P}\left(t-t^{\prime}\right)
$$

Sendo que P' é o total de chuva necessário para saturar o dossel. Considerando que não há gotejamento do dossel antes da saturação, assim P' também pode ser escrito como: 


$$
\left(1-p-p_{t}\right) P^{\prime}=S+\int_{0}^{t^{\prime}} E d t
$$

Considerando que não há gotejamento do dossel antes da saturação.

$$
I=\left(\frac{\bar{E}}{\bar{P}}\right) P+\left(S+\int_{0}^{t^{\prime}} E d t\right)\left\{1-\left(\frac{\bar{E}}{\bar{P}}\right)\left(1-p-p_{t}\right)^{-1}\right\}
$$

Equacionando, são identificados os coeficientes a e b da equação de regressão.

Sendo os valores de a e b derivados de uma regressão envolvendo um conjunto formado por diversos eventos de $\mathrm{P}$, seus valores são considerados representativos para qualquer evento de $\mathrm{P}$ no conjunto. Na maioria das regressões entre $I$ e $\mathrm{P}$ há grandes correlações, sugerindo que essa consideração fornece uma boa aproximação da realidade.

Segundo Gash (1979), infelizmente resultados de $I$ expressos em termos de $\mathrm{P}$ introduzem empirismos não apenas na taxa média da evaporação $\bar{E}$, mas também desnecessariamente na taxa média da precipitação $\bar{P}$. A duração da $\mathrm{P}$ é simples de se medir como na equação original, e de grande ajuda no entendimento dos estudos de $I$.

\section{RESULTADOS E DISCUSSÃO}

\subsection{Resultados observacionais}

Nas 40 coletas realizadas semanalmente durante março e dezembro de 2004, os totais de P e $I$ foram de $1.153,4 \mathrm{~mm}$ e $248 \mathrm{~mm}$, respectivamente $(\mathrm{I}=21,5 \%$ de $\mathrm{P})$.

Como descrito anteriormente, alguns parâmetros da vegetação local são necessários para a aplicação dos modelos de Rutter e de Gash, e foram obtidos das medidas realizadas em campo, que originaram modelos matemáticos. $\mathrm{O}$ valor do coeficiente de precipitação livre direta $(p=0,8127)$ foi obtido da equação de regressão linear PI $=0,8127(\mathrm{P})$ - 1,2993, com excelente coeficiente de determinação $\left(\mathrm{R}^{2}=0,9559\right)$. Enquanto que os valores da proporção de chuva que escoa pelos galhos e tronco $\left(\mathrm{p}_{\mathrm{t}}=0,0222\right)$ e da capacidade de armazenamento da superfície dos troncos $\left(\mathrm{S}_{\mathrm{t}}=0,1387\right)$ originaram-se da equação de regressão linear $\mathrm{ESC}=0,0222(\mathrm{P})-0,1387$ com $\mathrm{R}^{2}=0,5581$.

\subsection{Modelo de Rutter}

Como a região da FLONA de Caxiuanã apresenta uma grande quantidade de precipitação e esse conteúdo é dividido regularmente durante todo ano, com uma sazonalidade definida, o passo inicial para se estimar $I$ é o conhecimento dessa quantidade de água precipitada. Durante todo o ano de 2004, foram registrados cerca de $2.360 \mathrm{~mm}$ de precipitação considerando todos os eventos de $\mathrm{P}$ com qualquer volume de água.

Para a aplicação do modelo de Rutter é necessário se fazer várias considerações. Uma delas é a determinação da capacidade de armazenamento de água pelo dossel vegetal $\left(\mathrm{S}_{\mathrm{c}}\right)$, isto é, a quantidade de água que o dossel pode armazenar antes de atingir a saturação quando se inicia o gotejamento da água excedente. Para a FLONA de Caxiuanã o valor $\left(\mathrm{S}_{\mathrm{c}}=1,6\right.$ $\mathrm{mm}$ ) também foi obtido da equação de regressão linear PI = 0,8127(P) - 1,2993 com $\mathrm{R}^{2}=0,9559$. Desse modo, sabendo-se que o dossel atingiu a saturação com eventos de $\mathrm{P}>1,6 \mathrm{~mm} \mathrm{~h}^{-1}$, foram selecionados no grupo total de eventos de chuva somente aqueles superiores a esse valor.

Foram registrados 343 eventos durante todo o ano de 2004, que produziram um volume total de 2.019,4 mm de chuva (Figura 3), representando $85,6 \%$ da precipitação de todas as intensidades registradas no mesmo período.

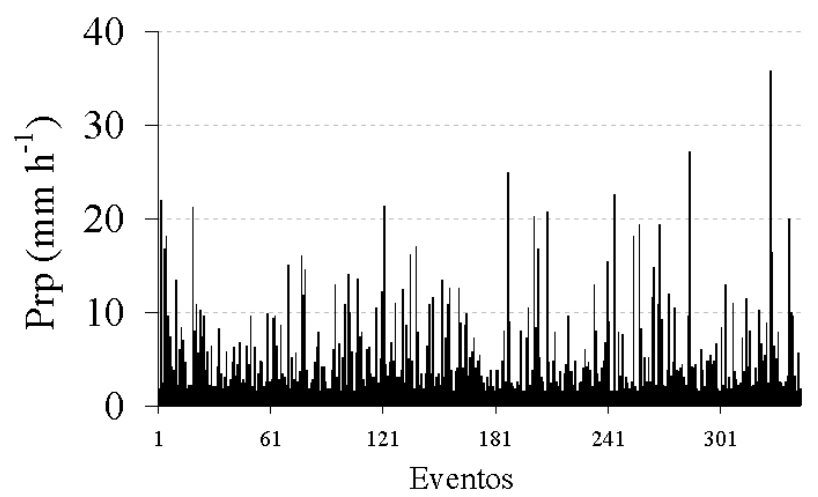

Figura 3 - Variação temporal da precipitação horária para eventos maiores que 1,6 $\mathrm{mm}$ por hora para a FLONA de Caxiuanã, durante todo o ano de 2004

A aplicação do modelo de Rutter com todas as considerações necessárias para as características específicas da FLONA de Caxiuanã e os parâmetros descritos anteriormente, resultou em uma estimativa de $I$ muito boa, quando se consideraram os totais acumulados da I medida e estimada. Entre 18 de março e 30 de dezembro de 2004, o modelo de Rutter superestimou $I$ desde o início até aproximadamente a metade do período (agosto), quando estimou $I$ com uma precisão melhor até meados de outubro, desse momento em diante, passou a subestimar $I$, até quase o final do período, quando eventos de $\mathrm{P}$ com grande intensidade ocorreram e provocaram um melhor ajuste do modelo de Rutter novamente.

O modelo de Rutter aplicado na FLONA de Caxiuanã gerou uma superestimativa de apenas $0,5 \%$ ou $1,1 \mathrm{~mm}$ da $I$ medida, para o total acumulado $(248 \mathrm{~mm})$ em quarenta coletas semanais. Essa excelente simulação para o valor acumulado, 
não significa que o modelo de Rutter simulou a $I$ tão bem para todos os dados semanais, visto que houve uma superestimativa mais acentuada durante o período de março a agosto, após esse período passou a subestimar os valores medidos. Em uma análise geral para todo o período estudado, o modelo de Rutter estimou $I$ de maneira excelente. A correlação entre os valores de $I$ medidos e estimados pelo modelo de Rutter, durante as quarenta semanas estudadas em 2004, apresentou ótimo coeficiente de determinação $\mathrm{R}^{2}=0,9829$ sendo realizada uma boa estimativa de $I$ (Figura 4).

Ubarana (1993) estudou o processo de interceptação da precipitação pelo dossel da floresta tropical úmida do tipo terra firme, na Reserva Florestal Ducke (RFD) no Amazonas. Aplicando o modelo de Rutter para a RFD o autor obteve a estimativa de $I(672 \mathrm{~mm})$ representando $12,9 \%$ da $\mathrm{P}(5.184 \mathrm{~mm})$ medida durante os dois anos, de 01 de setembro de 1983 a 31 de agosto de 1985. Usando os dados obtidos por Lloyd e Marques Filho (1988) durante o período de 03 de setembro de 1983 a 21 de agosto de 1985, proporcionalmente o modelo aplicado por Ubarana superestimou em $7,9 \%$ a I medida.

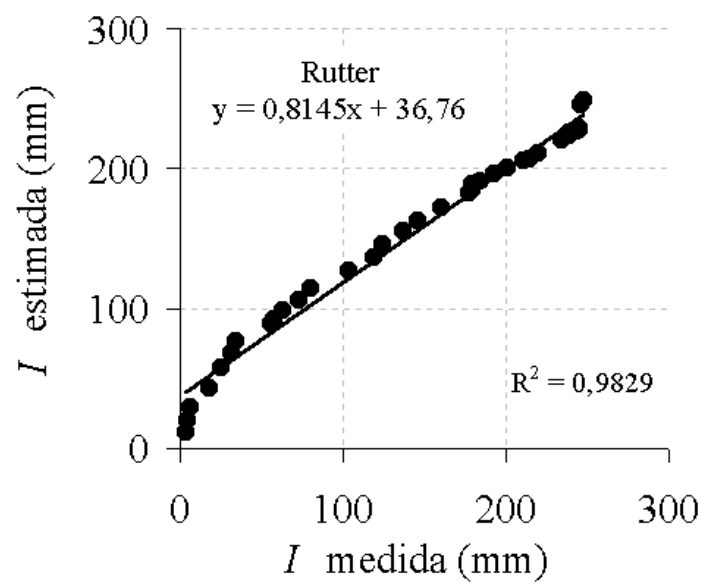

Figura 4 - Correlação entre os valores acumulados das interceptações medida e estimada pelo modelo de Rutter para a FLONA de Caxiuanã, entre março e dezembro de 2004

\subsection{Modelo de Gash}

Devido à impossibilidade de realização de medidas diárias, esses valores de diversos eventos de precipitação ocorridos durante a semana foram totalizados e então considerados como diários, o que possibilitou a determinação de um valor médio $\left(\bar{P}=1,2 \mathrm{~mm} \mathrm{~h}^{-1}\right)$, utilizados na entrada da modelagem de $I$. Valores de $\bar{P}$ para as quarenta semanas, entre março e dezembro de 2004, são apresentados na (Figura 5A), assim como os respectivos desvios-padrão.

Outra variável necessária como entrada do modelo é $\bar{E}$, nesse caso também foi considerado que cada período semanal de coleta representaria um dia, permitindo a determinaçã $\bar{E}$ de $=0,14 \mathrm{~mm} \mathrm{~h}^{-1}$.

Como o passo inicial para a seleção dos dados meteorológicos utilizados nos cálculos das estimativas de $I$ foi a escolha dos valores de $\mathrm{P} \geq 1,6 \mathrm{~mm} \mathrm{~h}^{-1}$, muitos eventos ocorreram durante o período noturno. Quando se calculou a evapotranspiração (E) para os quarenta períodos semanais, alguns valores de E foram negativos. Desse modo, para as estimativas de $I$ somente foram usados os dados de E positivos. Gash (1979) alertava para as dificuldades da utilização de valores empíricos como $\bar{P}$ e $\bar{E}$. No presente estudo, o valor de $\bar{E}=0,14 \mathrm{~mm} \mathrm{~h}^{-1}$ utilizado, está próximo do obtido por Souza Filho et al. (2005) que estudaram os mecanismos de controle da variação sazonal da evapotranspiração para o mesmo sítio experimental de Caxiuanã quando encontraram um valor de $\bar{E}=$ $0,15 \mathrm{~mm} \mathrm{~h}^{-1}$. A variação temporal de para as quarenta coletas semanais é apresentada na Figura 5B, assim como os respectivos desvios-padrão.

A

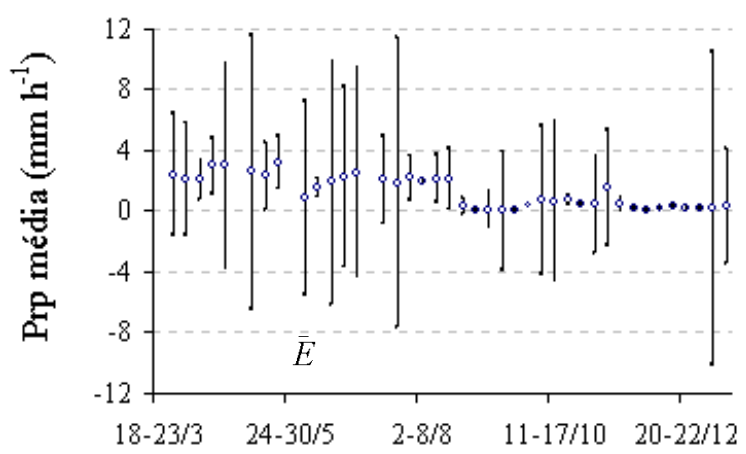

B

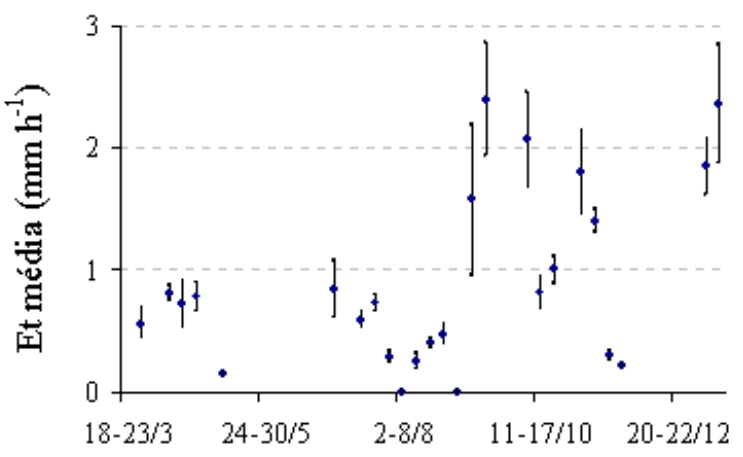

Figura 5 - Valores médios horários da Precipitação (A) e Evapotranspiração (B) para a FLONA de Caxiuanã, considerando-se chuvas maiores que $1,6 \mathrm{~mm} \mathrm{~h}^{-1}$, entre março e dezembro de 2004. As barras verticais são os desvios-padrão 
Assim como descrito por Gash (1979), as correlações entre os valores $\bar{E} \mathrm{e} \bar{P}$ não apresentaram bons coeficientes. Em seu estudo o autor encontrou valores de $\mathrm{R}^{2}=0,178$ e 0,008 para os anos de 1975 e 1976, respectivamente. No presente estudo para a FLONA de Caxiuanã, considerando apenas as coletas que apresentaram $\bar{E}$ positivos, o coeficiente de determinação encontrado foi $\mathrm{R}^{2}=0,196$. A relação $(\bar{E} / \bar{P})$ resultou em $\mathrm{a}=0,1156$.

Mesmo aplicando diversas considerações para se ajustar às características morfológicas da FLONA de Caxiuanã, o modelo simplificado de Gash apresentou satisfatória aproximação dos valores estimados e medidos da $I$ acumulada para o período de março a dezembro de 2004.

Para o total acumulado durante as quarenta coletas semanais entre março e dezembro de 2004, o modelo de Gash superestima $I$ em 42,8 mm (17,3\%). O modelo de Gash superestima os valores medidos de I no início (março e abril) e no final do período do estudado (dezembro), épocas em que há ocorrência de eventos de chuvas com grandes volumes. Para o período intermediário (de maio a novembro) o modelo subestima ou praticamente iguala os valores medidos. A correlação entre os valores medidos e estimados de $I$ (Figura 6) resultou na equação de regressão linear $\mathrm{y}=1,0084 \mathrm{x}+1,6296 \mathrm{com}$ um $\mathrm{R}^{2}=0,9621$.

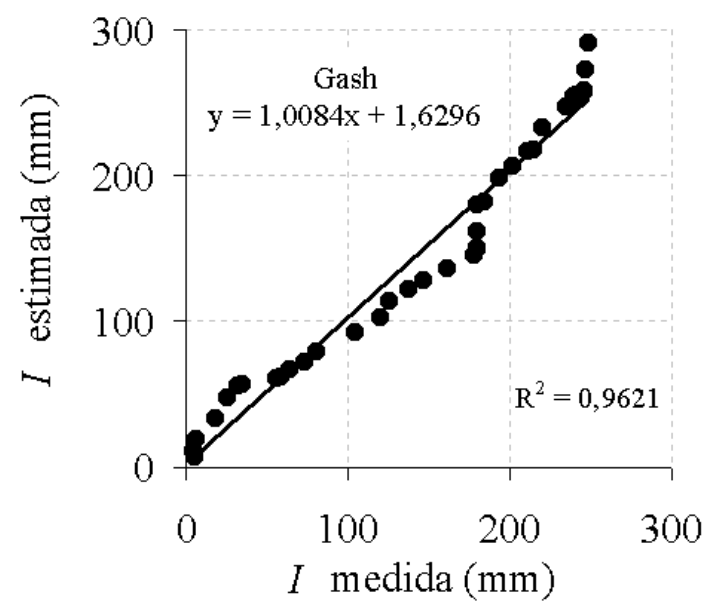

Figura 6 - Correlação entre os valores acumulados das interceptações medida e estimada pelo modelo de Gash para a FLONA de Caxiuanã, entre março e dezembro de 2004

\subsection{Comparação entre os modelos de Rutter e de Gash}

Grande parte dos estudos de $I$ realizados nas mais diferentes partes do mundo, e em distintos tipos de vegetação, objetiva encontrar uma maneira de determinar a quantidade de água representativa dessa componente importante do balanço hídrico. Fisch et al. (1998) reconhecem as dificuldades da realização de um balanço hídrico preciso para a região Amazônica, devido à descontinuidade espacial e temporal das medidas de precipitação, a inexistência de medidas simultâneas de vazões fluviais, o desconhecimento do armazenamento de água no solo etc.

A realização de medidas reais, in situ, nem sempre é possível. Devido às dificuldades financeiras ou de acesso físico aos locais de interesse, até a falta de informações meteorológicas básicas, e pessoal qualificado para realizar as coletas e o tratamento das informações. Essas e outras dificuldades existentes fazem com que, cada vez mais se procure aplicar modelos matemáticos na simulação da realidade de campo.

Como cada localidade de interesse para as pesquisas tem suas características peculiares, é normal que os modelos criados para determinada situação, não se mostrem produtivos para outras regiões.

Os modelos de Rutter e Gash escolhidos para as estimativas de $I$ na FLONA de Caxiuanã produziram bons resultados quando comparados aos valores obtidos em experimento observacional no campo (Figura 7).

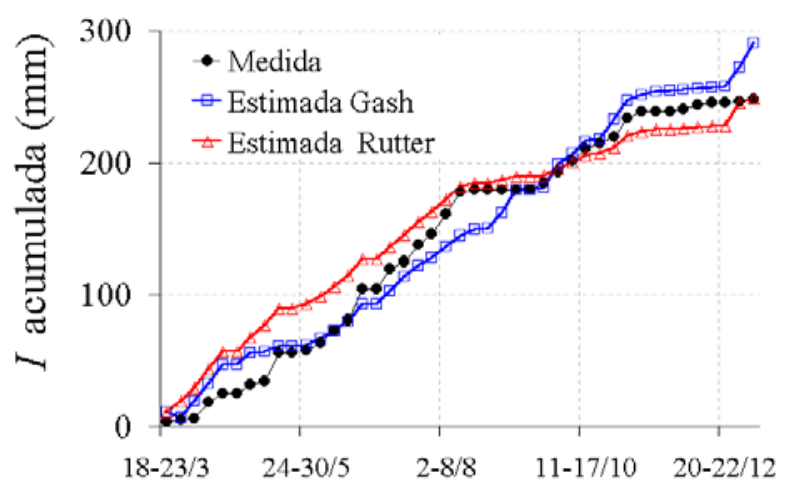

Figura 7 - Interceptação acumulada medida (círculos cheios), estimada pelo modelo de Gash (quadrados vazados) e estimada pelo modelo de Rutter (triângulos vazados) para a FLONA de Caxiuanã, entre março e dezembro de 2004

No presente estudo o modelo de Gash superestimou em $17,3 \%$ os valores medidos de $I$ ( $290 \mathrm{~mm}$ estimado e $248 \mathrm{~mm}$ medido), sendo esse resultado melhor comparativamente do que três estudos apresentados por Gash et al. (1995) e Valente et al. (1997), porém os referidos trabalhos foram realizados em florestas de pinheiros (Pinus pinaster) e eucalyptus globulus. Entretanto, só o tipo de vegetação não explica os resultados, pois em outra pesquisa para pinus sylvestris (Gash, 1979) obteve-se um valor simulado apenas $4,9 \%$ superior ao medido.

Na comparação da aplicação dos modelos de Gash e de Rutter em outras regiões (Tabela 1), observa-se que os resultados obtidos para a FLONA de Caxiuanã estão dentro de um intervalo aceitável de eficiência. 
O modelo de Gash também produziu melhores estimativas de I em um estudo desenvolvido por Zhang et al. (2005), que resultou em uma superestimativa de apenas 4,9\% para uma floresta subtropical mista na China. Esse modelo gerou o resultado mais próximo em uma floresta tropical na Indonésia, quando houve uma superestimativa de $14,4 \%$ do valor medido de $I$ (Asdak et al., 1998), esse resultado mostra, que possivelmente o modelo de Gash responda para florestas tropicais com superestimativas em torno dos $15 \%$ dos valores reais de $I$.

Na comparação dos resultados do modelo de Rutter, novamente o melhor resultado comparativo foi para a floresta tropical na Indonésia (Asdak et al., 1998) quando se superestimou $I$ em $6,4 \%$ do medido. Ubarana (1993) para a Reserva Florestal Ducke no Amazonas obteve 7,9\% aplicando esse modelo.

Tabela 1 - Comparação entre os resultados obtidos pela aplicação dos modelos de Rutter e Gash para estimativa de I

\begin{tabular}{|c|c|c|c|c|c|c|}
\hline \multirow[b]{2}{*}{ Modelos } & \multirow[b]{2}{*}{ Tịo de vegetação } & \multicolumn{2}{|c|}{$I(\mathrm{~nm})$} & \multicolumn{2}{|c|}{ Diferença } & \multirow[b]{2}{*}{ Autor } \\
\hline & & medida & estimada & (mm) & $(\%)$ & \\
\hline Futter & Floresta tropical & 623,1 & 672,2 & 49,1 & 7,9 & Ubarana (1993) \\
\hline Gash & Floresta tropical & 118,0 & 101,0 & 17,0 & 14,4 & Asdak et al.(1998) \\
\hline Rutter & Floresta tropical & 94,0 & 88,0 & 6,0 & 6,4 & Asdak et al. (1998) \\
\hline Gash & Floresta subtropical mita & 174,0 & 182,6 & 8,6 & 4,9 & Zhang et al. (2005) \\
\hline Gash & Floresta tropical & 247,97 & 290,78 & 42,8 & 17,3 & Este estudo \\
\hline Futter & Floresta tropical & 247,97 & 249,11 & 1,1 & 0,5 & Este estudo \\
\hline
\end{tabular}

\section{CONCLUSÕES}

É possível simular as perdas por interceptação da precipitação pela vegetação $(I)$ para a região tropical da FLONA de Caxiuanã, com bom nível de eficiência, utilizando os modelos de Rutter e Gash. O modelo de Gash gerou uma superestimativa de $I$ da ordem de $17,3 \%$ (42,8mm) do total acumulado (248 $\mathrm{mm})$ para o período de março a dezembro de 2004. Porém, durante as quarenta coletas semanais realizadas, esse modelo acompanhou satisfatoriamente as variações das medidas de $I$, entretanto sofreu a influência de dois grandes eventos de chuva registrados nas coletas do final do período, que aumentaram a estimativa acumulada.

Aaplicação do modelo de Rutter produziu superestimativas dos valores medidos de $I$ durante a maior parte do período analisado, entre março e setembro, passando a subestimar as medidas realizadas de outubro a dezembro de 2004. Para o total acumulado em todo estudo, o modelo de Rutter gerou uma superestimativa de apenas $0,5 \%(1,1 \mathrm{~mm})$ do valor de $I$ medido. Os resultados medidos na FLONA de Caxiuanã estão em concordância com outros estudos realizados que aplicaram os modelos de Rutter e Gash para a estimativa de $I$.

\section{AGRADECIMENTOS}

Os autores Leidiane Oliveira e Rafael Costa agradecem ao Conselho Nacional de Desenvolvimento Científico e Tecnológico - CNPq e à Coordenação de Aperfeiçoamento de Pessoal de Nível Superior - CAPES pelas bolsas de Pósgraduação. Agradecem também aos colegas envolvidos nas atividades de campo em Caxiuanã, em especial ao João Athaydes Jr. e ao Paulo Henrique Gonçalves.

\section{REFERÊNCIAS BIBLIOGRÁFICAS}

ASDAK, C; JARVIS, P.G.; GARDINGEN, P.V. Modelling rainfall interception in unlogged and logged forest areas of Central Kalimantan, Indonesia. Hydrol. Earth Sys. Sci. Discuss. vol. 2, n. 2-3, p. 211-220, 1998.

ELTAHIR, E.A.B.; BRAS, R.L. Precipitation recycling in the Amazon basin. Q. J. Roy. Meteor. Soc., vol. 120, n. 518, p.861-880, 1994.

FERREIRA DA COSTA, R.; COSTA, A.C.L. DA; MEIR, P.; MALHI, Y. ; BRAGA, A.P.; GONÇALVES, P.H.L.; SILVA JUNIOR, J.A.; SOTTA, E.D.; VALE, R.L.; GRACE, J.; 
FISHER, R.A. Projeto LBA/ESECAFLOR em Caxiuanã: características, atividades e resultados. In: Seminário de 10 anos de atividades da Estação Científica Ferreira Penna, Caxiuanã, Pará. Belém, 2003.

FIGUEROA, S.N. E NOBRE, C.A. Precipitations distribution over central and western tropical south America. Climanálise - Boletim de monitoramento e análise climática, v.5, n.6, pp. 36-48, 1990.

FISCH, G.; MARENGO, J.A.; NOBRE, C.A. Uma revisão geral sobre o clima da Amazônia. Acta Amazônica, v. 28, n. 2, p. 101-126, 1998.

GASH, J.H.C. An analytical model of rainfall interception by forests. Q. J. Roy. Meteor. Soc., vol. 105, n. 443, p. 43-55, 1979.

GASH, J.H.C.; LLOYD, C.R.; LACHAUD, G. Estimating sparse forest rainfall interception with an analytical model. J. Hydrol., vol. 170, p.79 - 86, 1995.

HORTON, R. E. Rainfall interception. Month. Weath. Rev., vol. 47, n. 9, p. 603-623, 1919.

LIMA, W.P; NICOLIELO, N. Precipitação efetiva e a interceptação em florestas de Pinheiros tropicais e em uma reserva de cerradão. Revista IPEF, n.24, p.43-46, ago, 1983.

LLOYD, C.R.; MARQUES FILHO, A.O. Spatial variability of throughfall and stemflow mensurements in Amazonian rainforest. Agric. For. Meteor., vol. 42, p.63-73, 1988.

MARENGO, J.A. Interannual variability of surface climate in the Amazon basin. Int. J. Climatol., v.12, Pp.853-863, 1992.

MORAES, J.C.; COSTA, J.P.R.; ROCHA, E.J.P. E SILVA, I.M.O. Estudos Hidrometeorológicos na Bacia do Rio Caxiuanã. In: Lisboa, P. L. B. (Org). Caxiuanã Cnpq/Museu Paraense Emílio Goeldi, Belém, p.85-95, 1997.

NOBRE, C.A.; SELLERS, P.J.; SHUKLA, J. Amazonian deforestation and regional change. J. Climate, vol. 4, p.957988, 1991.

OLIVEIRA, L.L. Avaliação da interceptação da precipitação pela vegetação na floresta nacional de Caxiuanã, Pará. 2007. 123p. Dissertação de Mestrado. Universidade Federal de Campina Grande - UFCG, Campina Grande, PB.

RUTTER, A.J., KERSHAW, K.A., ROBINS, P.C., MORTON, A.J. A predictive model of rainfall interception in forests, $\mathrm{i}$ derivation of the model from observations in a plantation of corsican pine. Agric. Meteor., vol. 9, p. 367-384, 1971.

RUTTER, A.J., MORTON, A.J., ROBINS, P.C. A predictive model of rainfall interception in forests, ii generalization of the model and comparison with observations in some coniferous and hardwood stands. J. Appl. Ecol., vol. 14, p. 567-588. 1975.
SILVA, A.S.L.; ALMEIDA, S.S.; ROSÁRIO, C.S. Flórula fanerogâmica da Estação Científica Ferreira Penna (ECFPN): Caracterização dos ecossistemas e lista preliminar de espécies. In: Seminário de 10 anos de atividades da Estação Científica Ferreira Penna, Caxiuanã, Pará. Belém, 2003.

SOUZA, E.P.P. Relações entre as anomalias de TSM do Atlântico e Pacífico e as precipitações na Amazônia oriental. 2003. 78p. Dissertação de mestrado. Instituto Nacional de Pesquisas Espaciais INPE, São José dos Campos, SP(INPE11435 - TDI/955).

SOUZA FILHO, J.D.C.; RIBEIRO, A.; COSTA, M.H.; COHEN, J.C.P. Mecanismo de controle da variação sazonal da transpiração de uma floresta tropical no nordeste da Amazônia. Acta Amazônica, v. 35(2), p. 223-229, 2005.

UBARANA, V.N. Experimentos observacionais e modelagem das perdas por interceptação da precipitação na floresta Amazônica. 1993. 115p. Dissertação de mestrado. Instituto Nacional de Pesquisas Espaciais INPE, São José dos Campos (INPE - 5583 - TDI/545).

VALENTE F.; DAVID, J.S.; GASH, J.H.C. Modelling interception loss for two sparse eucalypt and pine forest in central Portugal using reformulated Rutter and Gash analytical models. J. Hydrol., n190, p141-162, 1997.

VALENTE, F.M.R.T., Intercepção da precipitação em povoamentos florestais esparsos. Modelação do processo e características aerodinâmicas dos cobertos molhados. 1999. 172 p. Tese de Doutoramento em Engenharia Florestal, Instituto Superior de Agronomia, Universidade Técnica de Lisboa.

VIANA, J. S.; ALMEIDA, S. S.; CONCEIÇÃO, C.; FERREIRA, E.; ALVES, N.E SILVA, R. Comparação estrutural e florística entre os ambientes de terra-firme e igapó do entorno da Estação Científica Ferreira Penna - ECFPn. In: Seminário de 10 anos de atividades da Estação Científica Ferreira Penna, Caxiuanã, Pará. Belém, 2003.

ZHANG, G.; ZENG, G. M.; JIANG, Y. M.; HUANG, G. H.; LI, J. B.; YAO, J. M.; TAN, W; XIANG, R. J.; ZHANG, $X$. L. Modeling and measurement of two-layer-canopy interception losses in a subtropical mixed forest of centralsouth China. Hydrol. Earth Sys. Sci. Discuss. v.2, p. 1995-2024, 2005. 